\section{Reduced intensity conditioning haematopoietic \\ stem cell transplantation with mesenchymal \\ stromal cells infusion for the treatment of \\ metachromatic leukodystrophy: a case report}

\section{Dear Editor,}

In their letter, Charnas $L$ et al. are concerned about the accuracy of the diagnosis of metachromatic leukodystrophy (MLD) in our patient. ${ }^{1}$

Haematologica 2008; 93:e48 DOI: 10.3324/haematol.13015

First of all, we confirm that this case was discussed, as usual, in a multidisciplinary meeting including a neurologist, hematologists, a radiologist and the transplantation team. The procedure was approved by the hospital's Ethical Review Board.

The diagnosis was assessed by a neurologists' team as an adult form of MLD. This was based on the clinical symptoms, the symmetric predominantly white matter lesions (Figure 1) on the magnetic resonance imaging (MRI ) and the deficit in arylsulfatse A. Adult MLD has two major different clinical manifestations. One with a predominance of psychiatrics symptoms and impairment of intellectual functions ${ }^{2}$ and the second one with predominant motor impairment due to the neuropathy. ${ }^{3}$ In the reported case, the patient presented this second form. As usually described, we observed a decrease in the nerve conduction and an absence of the sensory potentials in the posterior tibial nerves. We did not perform a mutation analysis since it is recommended for the genetic screening of asymptomatic relatives.

Two other diagnoses could be made in this case: one is the multiple sulfatase deficiency (MSD) that combines clinical features of late infantile MLD and mucopolysaccharidosis. Patients with MSD present different degrees of skeletal anomalies, hepatosplenomegaly, deafness, cataract which usually suggest the problems associated with mucopolysacchaidosis. Our patient had none of those symptoms. The second disease that could be considered is sufatase activator deficiency (SAD) which may present the same clinical symptoms of MLD. However, in $\mathrm{SAD}$, the activity of arylsulfatase is normal and it was not the case in our patient.

In our article, we suggested the possible role of the transplantation in the stabilization of the clinical symptoms. Contrary to what the letter says, we did report the follow-up by MRI (see case report) that did not show any future deterioration of the cerebral lesions which correlates the promising clinical evolution.

We fully agree that such cases have to be collegially discussed and followed during and after their treatment. In the future, we will continue to follow this patient in a

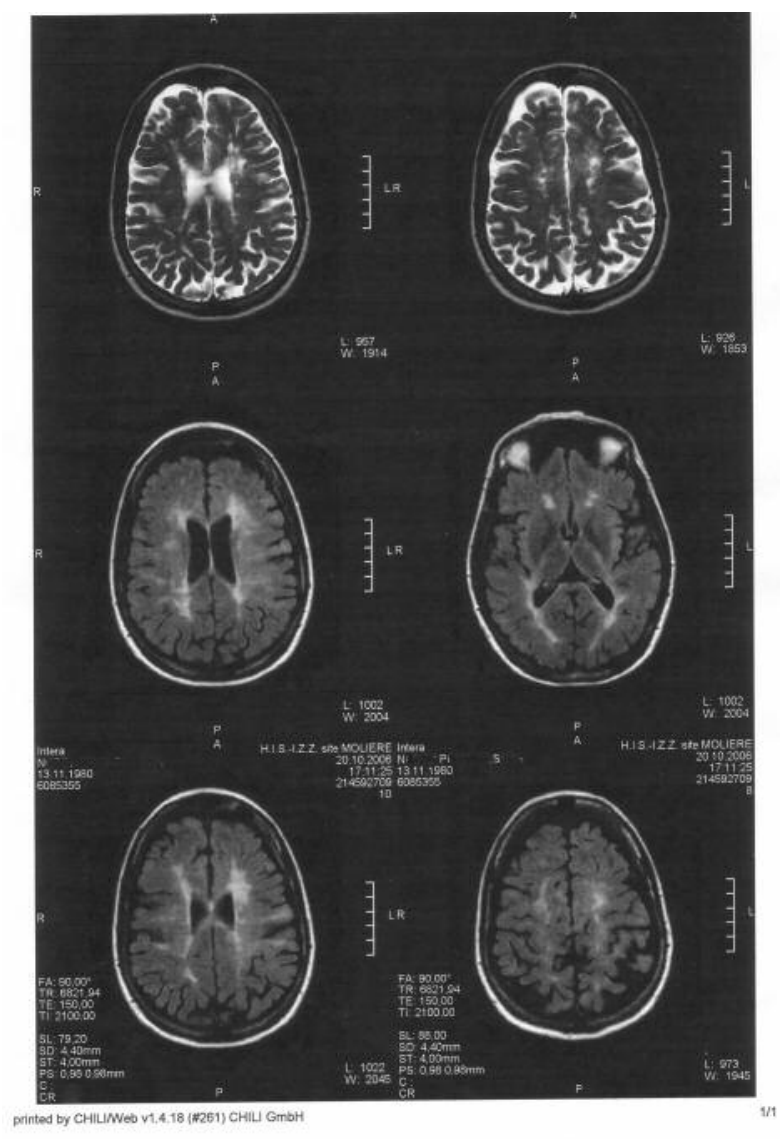

Figure 1. Magnetic resonance imaging of the patient showing typical changes throughout the white matter.

multidisciplinary approach as it has always been the case.

\section{Nathalie Meuleman,' Tatiana Tondreau, Geneviève Vanhaelen,' Laurence Lagneaux, ${ }^{,}$Dominique Bron \\ 'Department of Clinical and Experimental Hematology, Institut Jules Bordet, Université Libre de Bruxelles (ULB), Belgium; 'Department of Neurology, Centre Hospitalier Molière Longchamp, Brussels, Belgium.}

\section{References}

1. Meuleman N, Vanhaelen G, Tondreau T, et al. Reduced intensity conditioning haematopoietic stem cell transplantation with mesenchymal stromal cells infusion for the treatment of metachromatic leukodystrophy: a case report. Haematologica 2008;93:e11-3.

2. Black DN, Taber KH, Hurley RA. Metachromatic leukodystrophy: a model for the study of psychosis. J Neuropsychiatry Clin Neurosci 2003:15:289-93.

3. Fressinaud C, Vallat JM, Masson M, et al. Adult-onset metachromatic leukodystrophy presenting as isolated peripheral neuropathy. Neurology 1992;42:1396-8. 